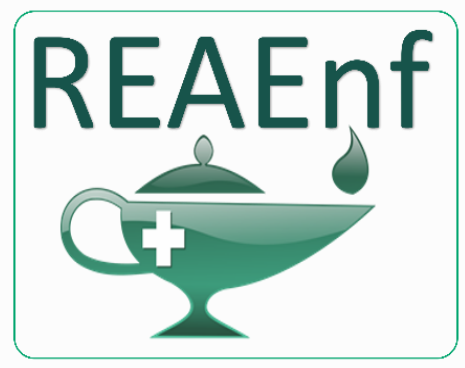

Revista Eletrônica Acervo Enfermagem
REVISÃO BIBLIOGRÁFICA

Recebido em: 9/2020

Aceito em: 10/2020

Publicado em: 11/2020

\title{
Assistência de enfermagem na segurança do paciente na UTI: uma revisão integrativa da literatura
}

\author{
Nursing assistance in patient safety at ICU: an integrative literature review
}

\begin{abstract}
Asistencia de enfermería en la seguridad del paciente en la UTI: una revisión bibliográfica integradora
\end{abstract}

Bárbara Alves Ruela de Azevedo Ruivo1, Jéssica Paloma Cardoso Bastos ${ }^{1}$, Adilson Mendes de Figueiredo Júnior ${ }^{2 *}$, Jamilly Cristina da Silva Silva ${ }^{2}$, Lorena Matos de Jesus², Glaucilene Viana Santa Brígida ${ }^{2}$, Christian Boaventura dos Santos ${ }^{3}$, Cristiane dos Santos Silva ${ }^{4}$, Edeiza Priscila de Souza Pinheiro ${ }^{5}$, Bianca Blois Pinheiro Camboim ${ }^{6}$.

Resumo: Este artigo tem como objetivo analisar a importância da assistência de enfermagem para a segurança do paciente em unidade de terapia intensiva. Esta é uma revisão integrativa da literatura. Nos resultados da análise do artigo, nota-se que os principais fatores que afetam a segurança do paciente são as condições do ambiente de trabalho da enfermagem, a atuação da equipe de enfermagem e os mecanismos efetivados para a segurança do paciente. Foi evidenciado que os principais erros e falhas que ocorrem estão relacionados aos medicamentos; aos eventos adversos observados, sobrecarga do profissional de enfermagem, relacionamento entre as equipes e a baixa continuidade da atenção prestada aos pacientes. Pode-se observar que a ocorrência de erros e eventos adversos dentro da UTI, em sua maioria, é da competência da enfermagem devido ao desempenho direto e constante do atendimento ao paciente. Assim, espera-se que este trabalho contribua para o entendimento da competência dos enfermeiros na UTI e da importância das práticas organizacionais, bem como para identificar possíveis fatores que contribuam para o envolvimento de possíveis falhas e erros na busca pela qualidade do atendimento.

Palavras-chave: Segurança do paciente, Unidades de terapia intensiva, Enfermagem.

Abstract: This article sought to analyze the importance of nursing care in patient safety in the Intensive Care Unit. It is an integrative literature review. Among the results found in the analysis of the articles, it was noticed that the main factors that affect patient safety, considered of greater relevance, are; the working conditions of the nursing team, the performance of the nursing team and the instruments used for patient

\footnotetext{
${ }^{1}$ Centro Universitário Maurício de Nassau (UNINASSAU), Belém - Pará.

2 Escola Superior da Amazônia (ESAMAZ), Belém - Pará. *E-mail: adilsonmdfj@hotmail.com

3 Universidade Federal do Pará (UFPA), Belém - Pará.

4 Universidade do Estado do Pará (UEPA), Belém - Pará.

${ }^{5}$ Centro Universitário do Pará (CESUPA), Belém - Pará.

6 Universidade da Amazônia (UNAMA), Belém - Pará.
} 
safety. It was evidenced that the main errors and failures that occur are related to drugs; the adverse events observed, overload of the nursing professional, relationship between the teams and the low continuity of care provided to patients. It can be observed that the occurrence of errors and adverse events within the ICU, for the most part, is the competence of nursing due to the direct and constant performance of patient care. Thus, it is expected that this work will contribute to the understanding of nurses' competence in the ICU and the importance of organizational practices, as well as to identify possible factors that contribute to the involvement of possible failures and errors in the search for quality of care.

Keywords: Patient safety, Intensive care units, Nursing.

Resumen: Este artículo buscó analizar la importancia del cuidado de enfermería en la seguridad del paciente en la Unidad de Cuidados Intensivos. Es una revisión integradora de la literatura. Entre los resultados encontrados en el análisis de los artículos, se notó que los principales factores que afectan la seguridad del paciente, considerados de mayor relevancia, son; las condiciones de trabajo del equipo de enfermería, el desempeño del equipo de enfermería y los instrumentos utilizados para la seguridad del paciente. Se evidenció que los principales errores y fallas que ocurren están relacionados con las drogas; los eventos adversos observados, sobrecarga del profesional de enfermería, relación entre los equipos y la baja continuidad de la atención brindada a los pacientes. Se puede observar que la ocurrencia de errores y eventos adversos dentro de la $\mathrm{UCl}$, en su mayor parte, es competencia de la enfermería debido a la realización directa y constante de la atención al paciente. Así, se espera que este trabajo contribuya a la comprensión de la competencia del enfermero en la UCl y la importancia de las prácticas organizacionales, así como a identificar posibles factores que contribuyan a la implicación de posibles fallas y errores la búsqueda de la calidad asistencial.

Palabras clave: Seguridad del paciente, Unidades de cuidados intensivos, Enfermería.

\section{INTRODUÇÃO}

No decorrer da ultima década com o avanço e aperfeiçoamento da ciência e da tecnologia a segurança do paciente tem sido uma preocupação constante na área da saúde em todas as suas dimensões no intuito de prestar uma assistência de qualidade e segura, principalmente nas áreas hospitalares de maior complexidade como na Unidade de Terapia Intensiva, que é considerado um ambiente de alto risco para a ocorrência de eventos adversa. Tendo em vista, que a segurança do paciente busca a redução do risco de um dano desnecessário associado ao cuidado de saúde (MENDES PSW, 2019).

A Unidade de Terapia Intensiva (UTI) se configura como uma unidade hospitalar restrita e complexa, dotada de sistema de monitorização contínua que tem como finalidade tratar os pacientes considerados graves e de alto risco e que muitas vezes possuem um estado crítico e necessitam de atendimento especializado e eficaz.

Dessa forma a assistência ofertada em uma UTI é considerada como sendo de alta complexidade, com isso, as chances de ocorrer eventos adversos e erros são ainda maiores, colocando em risco a segurança e a vida do paciente (MENDES PSW, 2019).

A interação das equipes interdisciplinares possui um trabalho sob pressão, pois, as intervenções integram a assistência à saúde, contribuindo para a prevenção de agravos à integridade física que ocasionam riscos de complicações e morte que resulta em normas e rotinas a serem seguidas para o bom funcionamento do serviço que é supervisionado pelo enfermeiro que está presente nas dimensões gerenciais e assistenciais do setor, com a finalidade de assegurar a qualidade de assistência de enfermagem; dimensionamento da equipe de enfermagem; gerenciar os recursos materiais e liderar a rotina da UTI (MAGALHÃES FHL, et al., 2019). O Enfermeiro possui um cargo cada vez maior de acordo com a demanda exigida pelo setor e dispõe de um papel vital para o seu bom funcionamento, que inclui entre eles a gestão, assistência e administrativo, que demanda do enfermeiro conhecimento prático-científico e o

REAEnf/EJNC | Vol. 5 | e5221 | DOI: https://doi.org/10.25248/REAenf.e5221.2020 Página 2 de 9 
domínio de suas competências privativas devido a ser competência do enfermeiro toda a organização da assistência ao cliente, sempre buscando meios para prestar uma assistência de qualidade e segurança para o paciente. Dentre todas as funções que o enfermeiro desempenha no ambiente hospitalar, a liderança tem sua maior importância (GOMES ATL, et al., 2019).

É de responsabilidade da Enfermagem proporcionar um ambiente tranquilo, e propício para o desenvolvimento do cuidado, livre de ruídos, tumultos e conversas paralelas; conhecer os requisitos básicos e necessários para manter o ambiente adequado para a realização dos procedimentos cirúrgicos e para a promoção dos cuidados necessários à promoção do conforto e bem-estar do cliente.

Tendo ainda, a autonomia e se valendo de suas ferramentas próprias de coleta de informações do paciente, tem a possibilidade de uma avaliação mais coesa e completa para intervenções que possam minimizar a ocorrência de lesões e danos para o paciente (GOMES ATL, et al., 2019). A assistência de enfermagem busca promover a saúde e o conforto, a preservação e a proteção da vida, implicando em ações tanto diretas quanto indiretas desenvolvidas para a recuperação e segurança do paciente. Neste cenário se entende que o enfermeiro, para desempenhar o seu trabalho na UTI, deva saber conduzir a equipe de enfermagem para obter o melhor resultado na assistência ao paciente (MAGALHÃES FHL, et al., 2019). Esse artigo buscou analisar a importância da assistência de enfermagem na segurança do paciente na Unidade de Terapia Intensiva.

\section{MÉTODOS}

O estudo trata-se de uma revisão integrativa da literatura que consiste em um método de pesquisa que permite buscar, analisar criticamente, e sintetizar evidências presentes na literatura acerca do tema pesquisado, resultando em um estudo representativo do estado do conhecimento atual da temática abordada, além disso, possibilita encontrarmos lacunas existentes no cenário cientifico que nos direcionam para o desenvolvimento de futuras pesquisar e intervenções. A realização desta revisão integrativa ocorreu em 6 etapas que consistiu na escolha do tema e as questões de pesquisa, estabelecimento de critérios para inclusão e exclusão de estudo, avaliação dos estudos incluídos, definição das informações a serem extraídas e utilizadas dos estudos selecionados, interpretação dos resultados e a síntese do conhecimento.

Nesta revisão, a coleta de dados foi realizada no período de Outubro a Dezembro de 2019, utilizando artigos e trabalhos científicos disponíveis na plataforma eletrônica Biblioteca Virtual em Saúde (BVS) que compõem as base de dados: Sistema Online de Busca e Análise de Literatura (PubMed), Literatura LatinoAmericana e do Caribe em Ciências da Saúde (Lilacs), Scientific Electronic Library Online (Scielo), Medical Literature Analysis and Retrievel System Online (Medline) e outras bases eletrônicas da área da saúde, utilizando a combinação dos seguintes descritores: segurança do paciente, unidades de terapia intensiva e enfermagem acrescido da palavra and. Tais descritores foram considerados como mais apropriados para a pesquisa após leituras previas de artigos abordando a temática pesquisada e a utilização da plataforma dos Descritores em Ciências da Saúde (DeCS).

Foram utilizados como critérios de inclusão para esta pesquisa os trabalhos científicos disponíveis na integra na internet, no idioma português do Brasil, inglês e espanhol, publicados no período de 2015 a 2019 e como critério de exclusão os trabalhos indisponíveis na integra, duplicados nas bases de dados, os que não correspondiam ou se enquadravam à temática abordada, os publicados fora do período determinado para a pesquisa e os que não respondiam a questão norteadora do estudo: Qual a importância da assistência de enfermagem na segurança do paciente na Unidade de Terapia Intensiva?

Após a análise dos critérios de inclusão e exclusão, a leitura do título e do resumo, foram selecionados 40 artigos para serem lidos na integra, no entanto, alguns deles não foram condicentes com a necessidade do estudo, resultando em 10 artigos selecionados, conforme observamos no Quadro 1.

Por fim, foi utilizado um instrumento de coleta de dados contendo a identificação do artigo (título, objetivo, método, pais e ano de publicação e principais resultados e recomendações/conclusões) com o intuito de facilitar e organizar o entendimento sobre o conteúdo dos artigos selecionados. 
Quadro 1 - Distribuição dos artigos científicos segundo base de dados e descritores. Belém, 2019.

\begin{tabular}{|c|c|c|c|}
\hline Descritores & $\begin{array}{l}\text { Base de } \\
\text { dados }\end{array}$ & $\begin{array}{c}\text { Artigos } \\
\text { encontrados }\end{array}$ & $\begin{array}{c}\text { Artigos } \\
\text { selecionados }\end{array}$ \\
\hline \multirow{4}{*}{$\begin{array}{l}\text { Segurança do paciente AND unidade de } \\
\text { terapia intensiva. }\end{array}$} & $\begin{array}{l}\text { PUBMED e } \\
\text { MEDLINE }\end{array}$ & 321 & 4 \\
\hline & LILACS & - & - \\
\hline & SCIELO & 125 & - \\
\hline & BDENF & 112 & 1 \\
\hline \multirow{4}{*}{$\begin{array}{l}\text { Segurança do paciente AND unidades de } \\
\text { terapia intensiva AND Enfermagem }\end{array}$} & $\begin{array}{l}\text { PUBMED e } \\
\text { MEDLINE }\end{array}$ & 71 & 3 \\
\hline & LILACS & 94 & - \\
\hline & SCIELO & - & - \\
\hline & BDENF & 90 & 1 \\
\hline \multicolumn{2}{|l|}{ Total } & 813 & 9 \\
\hline
\end{tabular}

Fonte: Ruivo BARA, et al., 2019.

\section{RESULTADOS}

Os dez artigos selecionados, três (30\%) foram publicados na língua portuguesa e sete $(70 \%)$ foram publicados na língua inglesa. A seguir serão apresentados os demais dados da amostra por meio do Quadro 2. Os elementos apresentados são o título do artigo, o nome dos autores, o ano de publicação, os objetivos e resultados bem como as conclusões de cada artigo. 
Quadro 2 - Relação dos artigos incluídos no estudo. Belém, 2019.

\begin{tabular}{|c|c|c|c|c|}
\hline Título do Artigo & Autor & Ano & Objetivo & Método \\
\hline $\begin{array}{l}\text { Associação entre clima de } \\
\text { segurança e a carga de } \\
\text { trabalho da enfermagem. }\end{array}$ & $\begin{array}{l}\text { De Souza VS, et al. } \\
\qquad(2019)\end{array}$ & 2019 & $\begin{array}{l}\text { Verificar a associação entre o clima de } \\
\text { segurança e a carga de trabalho dos profissionais } \\
\text { de enfermagem em uma Unidade de Terapia } \\
\text { Intensiva. }\end{array}$ & $\begin{array}{l}\text { Estudo transversal, analítico, } \\
\text { de abordagem quantitativa. }\end{array}$ \\
\hline $\begin{array}{l}\text { Elaboração e validação de } \\
\text { instrumento de assistência de } \\
\text { enfermagem para pacientes em } \\
\text { unidades de terapia intensiva }\end{array}$ & $\begin{array}{l}\text { Ferreira RC, et al. } \\
\qquad(2018)\end{array}$ & 2018 & $\begin{array}{c}\text { Elaborar e validar instrumento } \\
\text { para assistência de enfermagem, baseado } \\
\text { em literatura específica para pacientes } \\
\text { internados em Unidade de Terapia Intensiva, de } \\
\text { um hospital universitário do estado de São Paulo. }\end{array}$ & $\begin{array}{l}\text { Estudo metodológico para } \\
\text { construção e validação e um } \\
\text { instrumento }\end{array}$ \\
\hline $\begin{array}{l}\text { Segurança do paciente: } \\
\text { compreendendo o erro humano } \\
\text { na assistência de enfermagem } \\
\text { em terapia intensiva }\end{array}$ & $\begin{array}{l}\text { Duarte SCM, et al. } \\
\qquad(2018)\end{array}$ & 2018 & $\begin{array}{c}\text { Analisar as falhas ativas e as condições } \\
\text { latentes relacionadas a erros nos cuidados } \\
\text { intensivos de enfermagem e discutir as medidas } \\
\text { reativas e proativas mencionadas pela equipe de } \\
\text { enfermagem. }\end{array}$ & $\begin{array}{l}\text { Estudo qualitativo, descritivo e } \\
\text { exploratório. }\end{array}$ \\
\hline $\begin{array}{l}\text { Facilitated Nurse } \\
\text { Medication-Related Event } \\
\text { Reporting to Improve } \\
\text { Medication Management } \\
\text { Quality and Safety in Intensive } \\
\text { Care Units. }\end{array}$ & Xu J, et al. (2017) & 2017 & $\begin{array}{l}\text { O objetivo deste estudo foi explorar a utilidade } \\
\text { dos relatórios facilitados de ERM na identificação } \\
\text { de deficiências do sistema e a relação entre ERM } \\
\text { e o trabalho dos enfermeiros nas UTIs. }\end{array}$ & Estudo observacional \\
\hline
\end{tabular}




\begin{tabular}{|c|c|c|c|c|}
\hline $\begin{array}{c}\text { Evaluation procedures in } \\
\text { health: Perspective of nursing } \\
\text { care in patient safety. }\end{array}$ & $\begin{array}{c}\text { Da Costa TD, et al. } \\
(2017)\end{array}$ & 2017 & $\begin{array}{c}\text { A pesquisa objetivou analisar o cuidado de } \\
\text { enfermagem em unidades de terapia intensiva na } \\
\text { perspectiva da segurança do paciente, com base } \\
\text { na avaliação em saúde. }\end{array}$ & Estudo de avaliação \\
\hline $\begin{array}{c}\text { Incident and error reporting } \\
\text { systems in intensive care: a } \\
\text { systematic review of the } \\
\text { literature. }\end{array}$ & $\begin{array}{c}\text { Brunsveld-Reinders } \\
\text { AH, et al. (2016) }\end{array}$ & 2016 & $\begin{array}{c}\text { Avaliar (i) em que medida os sistemas de } \\
\text { notificação de incidentes (IRSs) na unidade de } \\
\text { terapia intensiva para adultos (UTI) atendem aos } \\
\text { critérios do Projeto de Diretrizes da OMS para } \\
\text { Sistemas de Aprendizagem e Comunicação de } \\
\text { Eventos Adversos, (ii) em que medida os IRSs } \\
\text { cumprem os quatro aspectos do ciclo da } \\
\text { qualidade iterativa e (iii) se os IRSs levaram a } \\
\text { medidas de melhoria na prática clínica }\end{array}$ & Revisão de literatura \\
$\begin{array}{c}\text { Percepção de } \\
\text { profissionais de enfermagem } \\
\text { acerca de segurança do } \\
\text { paciente em unidades de } \\
\text { terapia intensiva. }\end{array}$ & $\begin{array}{c}\text { Costa TD, et al. } \\
(2016)\end{array}$ & 2016 & $\begin{array}{c}\text { Compreender a percepção dos profissionais } \\
\text { de enfermagem nas unidades de terapia intensiva } \\
\text { dos hospitais públicos em relação à segurança do } \\
\text { paciente. }\end{array}$ & Estudo de natureza descritiva, \\
com abordagem qualitativa.
\end{tabular}

Fonte: Ruivo BARA, et al., 2019. 


\section{DISCUSSÃO}

Dentre os resultados encontrados da análise dos artigos, foi constatado no estudo de Capucho HC e Cassiani SHDB (2019); que quanto maior a carga horário do profissional de enfermagem, menor é a percepção e atuação sobre o clima de segurança do paciente. Já a pesquisa de THORNTON KC, et al. (2017) identificou o inverso, que uma forte cultura de segurança do paciente está associada a eventos adversos reduzidos, taxas de mortalidade mais baixas e custos mais baixos. Concomitantemente Duarte SCM, et al. (2018); identificou que os principais erros que afetam a segurança do paciente são ocasionados pela equipe de enfermagem, como a falta de checagem de medicações realizadas, falta de capacitação profissional, entre outras.

$\mathrm{Xu}$ J, et al. (2017); complementou que os eventos relacionados a medicamentos foram relatados em $35 \%$ das observações. O total de 60 eventos incluíram quatro erros de medicação e sete eventos adversos a medicamentos. Dos 49 eventos restantes, $65 \%$ foram associados ao impacto negativo do paciente. As deficiências da realização das tarefas e/ou processos foram o fator contribuinte mais comum para presença destes eventos. Porém, o autor ressaltou que a ocorrência destes foi correlacionada com o aumento do volume total de tarefas, como também o aumento da carga de trabalho, especialmente durante o turno da noite.

No resultado de Costa TD, et al. (2017); foi em relação a três elementos significativos, "comunicação e identificação", "terapia medicamentosa" e "higiene e conforto", mostrou o seguinte: 23 itens $(31,5 \%)$ foram considerados adequados e $50(68,4 \%)$, não conformes aos padrões exigidos para um atendimento confiável. Desses, 29 (39,7\%) foram classificados como parcialmente adequados e 21 (28,7\%) como inadequados, estabelecendo um cuidado preocupante no que se refere à segurança, com grande probabilidade de precipitação de eventos indesejáveis. Já Costa TD, et al. (2016); analisaram quatro classes: necessidade de mudança; ações antes de um evento adverso; conceitos de cuidados seguros; e ações que integram cuidados seguros. Foi evidenciado que a segurança do paciente é uma consequência da cultura adotada pelos profissionais.

No estudo sistematizado de Brunsveld-Reinders AH, et al. (2016); foram identificados um total de 2098 estudos, onde 36 estudos relataram o Sistema de Comunicação Incidentes (SCls) na UTI adulta. Os estudos foram divididos em: SCls específicos da UTI e SCls gerais. Os itens da lista de verificação da Organização Mundial da Saúde (OMS) foram avaliados e categorizados nas quatro fases do ciclo de qualidade iterativo.

Nenhum SCls cumpriu completamente os critérios da lista de verificação da OMS. Com relação ao loop iterativo, a entrada e a coleta de dados estão bem estabelecidas, mas pouca atenção foi dada à análise de incidentes e ao feedback. Isso resultou em um sistema de relatório administrativo, em vez do instrumento muito desejado para mudança de prática e aumento de qualidade, pois um SCls só pode efetivamente contribuir para melhorar a segurança do paciente e a qualidade do atendimento se mais atenção for dada à análise de incidentes e feedback.

Os esforços de melhoria da qualidade demonstraram ser mais eficazes e sustentáveis quando combinados com uma forte cultura de segurança. Existem diferentes metodologias para melhoria da qualidade da assistência em Unidade de Terapia Intensiva; uma abordagem criteriosa da implementação que envolva os profissionais de linha de frente e a liderança administrativa é essencial para o sucesso. Os esforços para incluir substancialmente pacientes e familiares nos processos de trabalho de melhoria da qualidade na UTI devem ser ampliados (THORNTON KC, et al., 2017).

Ferreira RC, et al. 2018; apontou que a maiorias dos instrumentos validados por enfermeiros estão diretamente relacionados à segurança do paciente, como por exemplo a higienização das mãos e manutenção das preocupações padrões.

Brunsveld-Reinders AH, et al. (2015); encontraram onze diretrizes existentes e cinco listas de verificação. Apenas uma lista de verificação cobriu as três fases: antes, durante e depois do transporte. As recomendações e os itens da lista de verificação concentraram-se principalmente na fase de pré-transporte. 
Os incidentes documentados mais frequentemente relacionados com a fisiologia do paciente e o mau funcionamento do equipamento e que ocorreram mais frequentemente durante o transporte. A discussão dos incidentes com médicos e enfermeiros da UTI resultou em recomendações importantes, tais como a introdução de uma lista de verificação padrão e a melhoria da comunicação com os outros departamentos.

Os incidentes sem lesão relacionados à competência da enfermagem, foco deste estudo, totalizaram 10.115 , ou seja $79,4 \%$ do total de incidentes sem lesão. As falhas no seguimento da prescrição da Enfermagem $(5.610,55,46 \%)$ foram os incidentes mais frequentemente observados neste estudo (Tabela 2).

Percebeu-se que os problemas abordados pelos profissionais hospitalares ou de atenção básica divergiam. No âmbito hospitalar, o maior problema referido foi a quantidade excessiva de medicações por paciente, intensificando a chance de erros na prescrição e administração de medicações. Já nas UBS houve mais relato de problemas no ambiente físico, visto que a maioria das unidades básicas não é planejada. Isso, na visão dos profissionais, gera tumulto nos atendimentos, sobrecarga dos profissionais e, consequentemente, facilita os erros de medicação (DIAS JD, et al., 2014).

Riscos de outras ordens também foram relacionados à insegurança do paciente pelos enfermeiros, tais como os institucionais, incluindo a sobrecarga de trabalho e as falhas na comunicação por parte dos membros da equipe: Como barreiras, foram destaques a falta de materiais, de manutenção de equipamentos, os cuidados não realizados devido à demanda excessiva, entre outros, conforme pode ser constatado nas falas dos enfermeiros (OLIVEIRA RM, et al., 2014).

A Enfermagem é a equipe que está prestando assistência direta ao paciente e sua atuação influencia positivamente ou não na implementação e qualidade da segurança do paciente. O estudo de Paiva MCMS, et al. (2011); aponta que um estudo realizado no estado de São Paulo identificou que os enfermeiros intensivistas entrevistados subnotificavam os Eventos Adversos (EAs) que acontecem em sua unidade, cerca de 50 a 71,4\%, sendo responsáveis principais por tal ação. Os entrevistados justificaram pela sobrecarga de trabalho, o esquecimento (não valorização do evento), o medo relacionado à cultura punitiva e a vergonha.

Complementado isto, a pesquisa de Nunes FDO, et al. (2014); apontou que 62,69\% dos técnicos de enfermagem apresentaram erros relacionados a medicação, destes $70 \%$ estava relacionado ao potencial de alteração a segurança microbiológica, $53,68 \%$ correspondiam ao preparo antecipado, esta prática modifica a composição química da medicação, alterando assim sua ação terapêutica e em 6,29\% houveram erro de dosagem. Moreira MD, et al. (2007); relataram que as quedas, apesar do percentual mínimo, são eventos que necessitam de cuidados, em especial com idosos. Visto que nos Estados Unidos da América esses EA instituíram a principal causa de mortes acidentais em idosos.

Diante das dificuldades e impossibilidades de descoberta, as pessoas buscam estratégias para mudar essa realidade e melhorar a segurança do paciente, como proporcionar condições suficientes para os funcionários que prestam cuidados diários aos pacientes; estimular os funcionários a usarem álcool em gel como forma eficaz de higienização das mãos e melhorar o treinamento e qualificação dos profissionais para que possam realizar as técnicas de higiene das mãos corretamente (NOVARETTI MCZ, et al., 2014).

Com o objetivo de consolidar um atendimento seguro e de qualidade, o Ministério da Saúde promulgou um decreto em 2013 que estipula a segurança dos pacientes nos serviços médicos. Isso inclui protocolos de prevenção de quedas; identidade do paciente; segurança das prescrições, uso e administração de medicamentos; cirurgia segura, práticas de higiene das mãos e úlceras de pressão (NUNES FDO, et al., 2014). Já em outro estudo, verificou-se a importância da utilização de checklists pela equipe nas salas cirúrgicas de um hospital de ensino (NOVARETTI MCZ, et al., 2014).

A cirurgia segura é um dos "desafios globais para a segurança do paciente", que propõe medidas de cuidado simples, como a confirmação de dados do paciente, informações clínicas e pessoais, a disponibilidade de todos os materiais e equipamentos que podem usar e o sucesso ou falha da operação normal. Essas verificações simples podem evitar uma série de complicações para o paciente. Ao mesmo 
tempo, este desafio e plano pode prevenir infecção do sítio cirúrgico, garantir anestesia segura, equipe cirúrgica segura e indicadores auxiliares cirúrgicos (PAIVA MCMS, et al., 2011).

\section{CONSIDERAÇÕES FINAIS}

Pode-se observar que as ocorrências de erros e eventos adversos dentro da UTI, em sua maioria, são de competência são de competência da equipe de enfermagem devido a realização direta e constante dos cuidados ao paciente e estão ligadas a medicamentos, falha na comunicação da equipe de enfermagem, falta de capacitação e sobrecarga do profissional, além de realização de procedimentos. Ainda há muito a percorrer nos caminhos da segurança do paciente, assim como também em aspectos que devem ser avaliados e prevenidos, como; a sobrecarga dos profissionais causada pelo excesso de trabalho ou devido a trabalho acumulado, a ausência de diálogo entre a equipe de enfermagem, a falta de entendimento entre a equipe multiprofissional, e a não realização do checklist recomendado pela OMS.

\section{REFERÊNCIAS}

1. BRUNSVELD-REINDERS $\mathrm{AH}$, et al. A comprehensive method to develop a checklist to increase safety of intrahospital transport of critically ill patients. Critical Care, 2015; 19(1): 1-10.

2. BRUNSVELD-REINDERS AH, et al. Incident and error reporting systems in intensive care: a systematic review of the literature. International Society for Quality in Health Care, 2016; 28(1): 2-13.

3. CAPUCHO HC e CASSIANI SHDB. Necessidade de implantar Programa de segurança do paciente no Brasil. Revista de Saude Publica, 2013; 47(4): 791-798.

4. COSTA TD, et al. Percepção de profissionais de enfermagem acerca de segurança do paciente em unidades de terapia intensiva. Revista Gaucha de Enfermagem, 2016; 37(3).

5. DE SOUZA VS, et al. Associação entre clima de segurança e a carga de trabalho da enfermagem. Cogitare Enfermagem, 2019; 24(0).

6. DA COSTA TD, et al. Evaluation procedures in health: Perspective of nursing care in patient safety. Applied Nursing Research, 2017; 35(1): 71-76.

7. DIAS JD, et al. Compreensão de enfermeiros sobre segurança do paciente e erros de medicação. REME: Revista Mineira de Enfermagem, 2014; 18(4), 866-880.

8. DUARTE SCM, et al. Segurança do paciente: compreendendo o erro humano na assistência de enfermagem em terapia intensiva. Revista Da Escola de Enfermagem Da USP, 2018; 52(1).

9. FERREIRA RC, et al. Elaboração e validação de instrumento de assistência de enfermagem para pacientes em unidades de terapia intensiva. Cogitare Enfermagem, 2018; 23(4).

10. GOMES ATL, et al. Safety of the patient in an emergency situation: perceptions of the nursing team. Revista Brasileira de Enfermagem, 2019; 72(3): 753-759.

11. MAGALHÃES FHL, et al. Clima de segurança do paciente em um hospital de ensino. Revista Gaucha de Enfermagem, 2019; 40(1).

12. MENDES PSW. Segurança do paciente: conhecendo os riscos nas organizações de saúde [online] (2nd ed.). 2019.

13. MOREIRA MD, et al. Variáveis associadas à ocorrência de quedas a partir dos diagnósticos de enfermagem em idosos atendidos ambulatorialmente. Revista Latino-Americana de Enfermagem, 2007; 15(2): 311-317.

14. NOVARETTI MCZ, et al. Nursing workload and occurrence of incidents and adverse events in ICU patients. Revista Brasileira de Enfermagem, 2014; 67(5): 692-699.

15. NUNES FDO, et al. Segurança do paciente: como a enfermagem vem contribuindo para a questão? Revista de Pesquisa Cuidado é Fundamental Online, 2014; 6(2): 841-847.

16. OLIVEIRA RM, et al. Estratégias para promover segurança do paciente: da identificação dos riscos às práticas baseadas em evidências. Escola Anna Nery - Revista de Enfermagem, 2014; 18(1): 122-129.

17. PAIVA MCMS, et al. Eventos adversos: análise de um instrumento de notificação utilizado no gerenciamento de enfermagem. Revista Da Escola de Enfermagem, 2011; 44(2): 287-294.

18. THORNTON KC, et al. Preventing Harm in the ICU - Building a Culture of Safety and Engaging Patients and Families. Critical Care Medicine, 2017; 45(9): 1531-1537.

19. XU J, et al. Facilitated Nurse Medication-Related Event Reporting to Improve Medication Management Quality and Safety in Intensive Care Units. Nursing Research, 2017; 66(5): 337-349. 